\title{
Genetic and functional analysis of the bovine uterine microbiota. Part I: Metritis versus healthy cows
}

\author{
M. L. S. Bicalho, V. S. Machado, C. H. Higgins, F. S. Lima, and R. C. Bicalho ${ }^{1}$ \\ Department of Population Medicine and Diagnostic Sciences, College of Veterinary Medicine, Cornell University, Ithaca, NY 14853
}

\begin{abstract}
Metritis is a uterine disease that affects 10 to $30 \%$ of all lactating dairy cows and has detrimental effects on reproductive performance, milk production, and survival. Data regarding the identity and abundance of bacterial genes governing traits such as virulence, antibiotic resistance, and stress responses could enable identification of previously unknown agents that play a role in metritis pathogenesis. Moreover, such knowledge could lead to the development of improved treatments or preventive methods. Therefore, the objectives of this study were to characterize the uterine microbial population and to differentiate, for the first time, the microbial functional diversity in cows with metritis versus healthy cows. In addition, we aimed to identify relationships between microbial genes and postpartum uterine health. Uterine swabs were collected from 24 cows within 3 to $12 \mathrm{~d}$ in milk; 12 cows were diagnosed with metritis and the other 12 were healthy. Metritis was defined as a watery, reddish or brownish uterine discharge having a fetid smell, and rectal temperature greater than $39.5^{\circ} \mathrm{C}$. Cows with a clear and viscous uterine discharge, not fetid or mucopurulent, were classified as healthy. Microbial metagenomic DNA from uterine swab samples was subjected to whole-genome shotgun sequencing on the Illumina MiSeq platform (Illumina Inc., San Diego, CA). The MG-RAST server (metagenomic rapid annotations using subsystems technology; http://metagenomics.anl.gov/) and STAMP software (http://kiwi.cs.dal.ca/Software/STAMP) were used to detect statistically significant differences in the abundance of taxonomic and functional features between the uterine microbial metagenomes of metritic and healthy cows. Our results showed an increased abundance of Fusobacteria and Bacteroidetes in metritic cows, confirming the potential role of those 2 taxa in the pathogenesis of metritis. The MG-RAST analysis revealed a significantly higher abundance of genes for protein transport across the cytoplasmic membrane and type VI
\end{abstract}

Received September 27, 2016.

Accepted January 14, 2017.

${ }^{1}$ Corresponding author: rcb28@cornell.edu bacterial secretion systems in the metritic microbiota. Additionally, genes coding for resistance to acid stress were exclusive to the metritis microbiota, suggesting that microbial resistance to acid stress is important for microbial survival in the infected uterus. On the other hand, genes coding for adhesion molecules, bacteriocins, and antibacterial peptides were significantly associated with the uterine microbiota of healthy cows, as was tolerance to colicin E2.

Key words: metritis, shotgun, microbiome

\section{INTRODUCTION}

Metritis is highly prevalent in dairy cows and is one of the most important diseases in the dairy industry worldwide, causing economic losses due to lower conception rates, premature culling, increased use of antibiotics, reduced milk production, and treatment costs (Drillich et al., 2001; Sheldon et al., 2006). In addition, metritis is a serious animal welfare issue, and affected cows have been shown to experience severe pain (Stojkov et al., 2015). In North America, metritis affects 10 to $30 \%$ of all lactating dairy cows (Bicalho et al., 2010; LeBlanc et al., 2011; Lima et al., 2014).

The multifactorial etiology of metritis presents a major challenge for disease prevention and treatment. Intrauterine microbial contamination is ubiquitous shortly after parturition, and certain microbial profiles are associated with disease whereas others are not (Sheldon et al., 2006; Santos and Bicalho, 2012; Jeon et al., 2015). Therefore, understanding the dynamics of the uterine microbiota at parturition is essential when developing effective treatment strategies that aim not only toward clinical cure, but also to facilitate the process of restoring a healthy uterine microbiota status.

Culture-independent methods to analyze microbial populations have become increasingly sophisticated, and most of the latest technologies have been applied to investigate microbial communities in the bovine uterus (Machado et al., 2012; Santos and Bicalho, 2012; Jeon et al., 2015). The first description of the diversity of bacterial populations in the uterine microbiota by cultureindependent analyses showed that the complexity and assemblage of the intrauterine bacterial community in 
postpartum cows differs dramatically between healthy cows and metritic cows (Santos et al., 2011). Importantly, Escherichia coli has been repeatedly described as the primary pathogen responsible for initiating postpartum uterine infection or inflammation, disrupting the natural balance of the uterine microbiota to favor the appearance of opportunistic anaerobic bacteria; for example, Fusobacterium necrophorum and Bacteroides spp. (Bonnett et al., 1991; Bicalho et al., 2010; Sheldon et al., 2010).

Recent studies have made use of next-generation sequencing of the bacterial $16 \mathrm{~S}$ ribosomal DNA to describe the microbial composition of the postpartum uterine environment of dairy cows (Santos et al., 2011; Machado et al., 2012; Santos and Bicalho, 2012; Jeon et al., 2015). Comparative analysis of the sequences of the $16 \mathrm{~S}$ rDNA gene from different species is a powerful way to describe the phylogenetic composition of complex bacterial communities; however, this technique cannot provide any information regarding the genetic functional potential of the studied bacterial communities. Shotgun metagenomics avoids PCR bias and is not restricted to bacterial sequences. It provides an alternative approach to study the non-cultivable microbiota, offering a wider perspective on microbial diversity, including DNA viruses and yeasts. In addition, the coverage of the genome outside of the small 16S rDNA gene region means that specific, strain-level discrimination is achievable (Hilton et al., 2016).

Ultimately, due to the high pressure on the use of antibiotics, especially in food animals, and the high demand for new therapeutic strategies, the next steps of microbiome research should focus on its application to prevention science. Although metagenomic sequencing is much more expensive than $16 \mathrm{~S}$ sequencingespecially to achieve the coverage and depth needed for species identification - and a common weakness for high-throughput sequencing methods is database bias, as sequencing becomes more widely used, databases are growing in size and diversity. Additionally, the greatest drawback of this approach is the noisy signal due to host contamination, which can deluge the signal from pathogens (as we have seen in our data), which may lead to an inaccurate classification of the pathogenic community (Kuczynski et al., 2011). However, because uncovering the microbial communities of distinct niches of the body is an important component of detection of patterns and understanding the effects of the microbiome on animal health, any achievement of microbiome research and the potential use of metagenomic tools to prevent disease and to detect risk factors for disease development to the diagnostic process would have an immediate effect on animal health and production.
Furthermore, by knowing the functional potential of the metagenome, we can infer what the microbial community is capable of doing (Roossinck et al., 2015). For example, the metagenome of a complex microbial community (e.g., human feces) has been reported to be linearly correlated with the metatranscriptome, indicating that the measured functional potential and the activity of the microbiome share many similarities (Franzosa et al., 2014). Thus, generating data regarding the identity and abundance of genes related to virulence, metabolism, and antibiotic resistance could enable identification of unknown etiological agents that play a role in metritis pathogenesis. In addition, alternative treatments or preventive methods could be developed.

To date, the bovine intrauterine microbiome has not yet been studied using shotgun metagenomics. Therefore, the objectives of this study were (1) to characterize the uterine microbial population and its functional diversity in healthy cows and cows affected with metritis; and (2) to identify relationships between microbial genes and postpartum uterine health.

\section{MATERIALS AND METHODS}

\section{Ethics Statement}

This study was carried out in strict accordance with the recommendations of The Animal Welfare Act of 1966 (AWA) (P.L. 89-544) and its amendments, 1970 (P.L. 91-579), 1976 (P.L. 94-279), and 1985 (P.L. 99198), that regulate the transportation, purchase, care, and treatment of animals used in research. The research protocol was reviewed and approved by the Institutional Animal Care and Use Committee of Cornell University (protocol number 2011-0111). Sampling procedures and experimental manipulations were authorized by the farm owner, who was aware of the procedure. No specific permissions were required for these locations or activities. We confirm that the field of the study did not involve endangered or protected species.

\section{Farm Management and Facilities}

Samples were collected from a single farm located near Ithaca, New York, from June 7 to 14, 2014. This farm milked approximately 4,000 cows, and the herd consisted of Holstein cows housed in freestall barns with concrete stalls covered with rubber mattresses and bedded with sand. After parturition, cows were moved from the maternity pen to a fresh cow pen (freestall barn) and kept in the same pen until approximately 20 DIM. This pen was monitored daily by farm employees, 
and cows were submitted for a complete physical exam if they showed signs of dullness and depression.

\section{Case Definition}

Cows within 3 to 12 DIM were evaluated for vaginal discharge for the diagnosis of metritis by one of the veterinarians of the research team. Vaginal discharge was obtained by retracting the cervix and uterus to obtain fluid of uterine origin through rectal manipulation. $\mathrm{Pu}-$ erperal metritis was clinically defined as an abnormally enlarged uterus and a fetid, watery, red-brown uterine discharge, with signs of systemic illness (decreased milk yield, dullness, and other signs of toxemia) and rectal temperature greater than $39.5^{\circ} \mathrm{C}$ (Sheldon et al., 2006). Metritic cows that had been recently treated with antibiotics were excluded from sample collection. Cows with a clear and viscous uterine discharge, not fetid or mucopurulent, were classified as healthy. Healthy and metritic cows were matched on the day of diagnosis and paired by DIM and parity.

\section{Sample Collection}

For metagenomics sequencing, uterine swabs were collected from 24 cows, 12 diagnosed with metritis and 12 healthy. Briefly, cows were restrained and the perineum area was cleansed and disinfected with $70 \%$ ethanol. Then, a sterile swab (Double-Guarded Uterine Culture Swab, Har-Vet McCullough, Spring Valley, WI) covered by a sterile pipette (inside a plastic sheath) was introduced to the cranial vagina. To avoid vaginal contamination of the swab, the plastic-sheath-covered pipette was directed into the cervix; inside the cervix, the plastic sheath (first layer of protection) was ruptured and the pipette was then manipulated through the cervix into the uterus. Once inside the uterus, the swab was advanced through the sealed plastic pipette (second layer of protection), exposing the sterile cotton swab to uterine secretion. Before removal, the swab was pulled back inside the pipette while the pipette was still inside the uterus to avoid contamination by vaginal fluid. Each swab was transferred to a $15-\mathrm{mL}$ conical sterile polypropylene centrifuge tube, placed on ice, transported to the laboratory within $4 \mathrm{~h}$, and then frozen at $-80^{\circ} \mathrm{C}$.

\section{DNA Extraction}

Before DNA extraction, swab samples were transferred to a DNA-/RNA-free 2-mL microcentrifuge tube and immersed in $1.5 \mathrm{~mL}$ of DNA-/RNA-free water and settled in a Mini-Beadbeader-8 (Biospec Products, Bartlesville, OK) to disperse any mucus, bacteria, or cells. Swabs were removed from the tubes and the suspension was centrifuged at $10,000 \times g$ for $10 \mathrm{~min}$. The supernatant was discarded and the pellet was used for extraction of total DNA, which was performed using a PowerSoil DNA Isolation Kit (MO BIO Laboratories Inc., Carlsbad, CA) following the manufacturer's recommendations. The concentration of DNA was evaluated using a Quant-iT PicoGreen dsDNA Assay Kit (Life Technologies Corp., Carlsbad, CA).

\section{Whole-Metagenome Shotgun Sequencing}

An aliquot of each extracted sample was normalized to a concentration of $0.2 \mathrm{ng} / \mu \mathrm{L}$. After normalization, the samples were processed by using a Nextera XT DNA Sample Prep Kit (Illumina Inc., San Diego, CA). Tagmentation of the samples was done using $1 \mathrm{ng}$ of template, as directed by the manufacturer. Following tagmentation, PCR amplification was carried out according to the manufacturer's instructions using a unique combination of indexing primers for each of the 21 samples, allowing the samples to be multiplexed. Three samples from healthy cows were discarded from further processing and analysis due to low quality. Following amplification, short DNA fragments were removed from each library using AMPure XP (Beckman Coulter Inc., Indianapolis, IN) bead purification, and the resulting libraries were then normalized using Library Normalization (Nextera XT DNA LibraryPrepReference Guide; Illumina Inc.) beads/additives. In addition, to prepare for cluster generation and sequencing, equal volumes of normalized libraries were combined, diluted in hybridization buffer, and heat denatured, according to the Nextera XT protocol. Finally, pair-end sequencing was performed using a MiSeq Reagent Kit v3 (600 cycles) through the Illumina MiSeq platform (Illumina Inc.).

\section{Bioinformatics}

Raw data files were de-multiplexed and converted to fastq files using Casava v.1.8.2 (Illumina Inc.). Fastq files were concatenated and uploaded to the MG-RAST server (metagenomic rapid annotations using subsystems technology; Meyer et al., 2008) to determine relative abundance of the microbiota at the phylum, genus, and species levels, and to predict functional abundance. Through MG-RAST analysis, sequences were subjected to quality control, which included dereplication (removal of sequences produced by sequencing artifacts), removing host-specific sequences (Bos taurus, UMD v3.0; ftp://ftp.cbcb.umd.edu/pub/data/Bos_taurus/ latest_version/README.txt), ambiguous base filtering (removing sequences with $>5$ ambiguous base pairs), 
and length filtering (discarding sequences whose length differs from the average sequence length by more than the specified number of standard deviations of length). The MG-RAST analysis uses a 2-step approach for feature identification: FragGeneScan (FGS) and a similarity search for ribosomal RNA against a nonredundant integration of the Silva (Pruesse et al., 2007), Greengenes (DeSantis et al., 2006), and RDP databases (Cole et al., 2009). The non-redundant multi-source protein annotation database (M5NR; Wilke et al., 2012) was the annotation source used for phylogenetic classification, with a minimum e-value of $1 \times 10^{-5}$, a minimum identity of $90 \%$, and a minimum alignment length cutoff of 15. Subsystems under SEED (www.theseed.org/) were used for functional annotation analysis. The predicted functional profile analysis was performed using hierarchical classification, with a maximum e-value of $1 \times 10^{-5}$, a minimum identity of $90 \%$, and a minimum alignment length cut-off of 15 .

\section{Statistical Analysis}

Sequencing reads from the DNA libraries were used to determine the phylogenetic composition and functional distribution of the respective health status groups (i.e., metritis or healthy). Each value obtained indicated the relative (percentage) frequency of reads with predicted proteins and ribosomal DNA genes annotated to the indicated taxonomic level. This analysis eliminates any interference of phyla that were highly abundant and allows the visualization of less abundant (or rare) groups.

The differentially abundant taxa and SEED functions were identified using Statistical Analysis of Metagenomic Profiles (STAMP; http://kiwi.cs.dal. ca/Software/STAMP) software as previously described by Parks et al. (2014). The phylogenetic table of metagenomes comprising abundance profiles at each taxonomic level based on the "best hit classification" method was imported into the STAMP tool. Taxonomic data were analyzed at the phylum and genus levels selective to any single metagenome sample. Graphs of taxon mean relative abundance were constructed in Excel (Microsoft Corp., Redmond, WA). Similarly, a functional abundance profile based on "hierarchical classification" was generated for the metagenome data set and subsequently imported into STAMP. For the functional abundance profile, the statistical tests were applied at level 1 of functional annotations. Statistical analysis using Welch's test with Benjamin-Hochberg false discovery rate correction was applied to these data sets to derive statistically significant differential features between the 2 metagenomes. Extended error bar plots were generated for bacterial genera and annotated functions common to metritic and healthy cows to illustrate dissimilarities in mean proportion and their differences in the 2 data sets. The results were filtered using a $P$-value of 0.05 and an effective size of 0.5 thresholds in STAMP. Further analysis was carried out on categories within filtered functional subsystems for statistical significance based on analysis of similarity (ANOSIM) using Paleontology Statistics (PAST) software (Hammer et al., 2001). The value of ANOSIM statistic R close to 1.0 suggests dissimilarity between groups, whereas an $R$ value close to 0 suggests an even distribution of high and low ranks within and between groups. Any $\mathrm{R}$ values below 0 suggest that dissimilarities are greater within groups than between groups (Clarke and Gorley, 2001).

\section{RESULTS}

\section{Sequencing Results}

Metagenome sequencing of 20 samples resulted in a total of 7.4 million raw reads and approximately 6.3 million reads after quality filtering. One sample from a cow with metritis were excluded from further analysis due to low number of sequences reads (contains 23,802 sequences). The sequencing data of the individual samples are detailed in Supplemental Table S1 (https://doi. org/10.3168/jds.2016-12058). Contingent on the MGRAST annotation, about 0.1 to $0.5 \%$ of the sequences that passed quality control were classified as rDNA per uterine microbiome sample based on the hits against $16 \mathrm{~S}$ rDNA gene sequence databases (hits represent the number of times a particular taxon is detected), whereas 75 to $95 \%$ of the reads from all samples predicted protein-coding regions. We obtained a combined total of approximately 2,960,774 high-quality bacterial reads that were classified into various functional subsystems, and 25,334 high-quality $16 \mathrm{~S}$ rDNA sequence reads. The rDNA-similar reads were clustered at $97 \%$ identity, and the longest sequence was picked as the cluster representative.

To assess the depth of sequencing performed for each sample, comparative rarefaction curves were generated using MG-RAST. Rarefaction analysis showed that, for some samples (18), the number of sequences screened was sufficient to reveal the total number of sequence types within these samples, as they reached a plateau (data not shown). For other samples (2), the rarefaction curves showed that more sequences would be necessary to capture all sequence types present to reflect the complete diversity within the sample. Interestingly, the samples that showed the steepest rarefaction curves, with the exception of sample number 4613438.3 from 

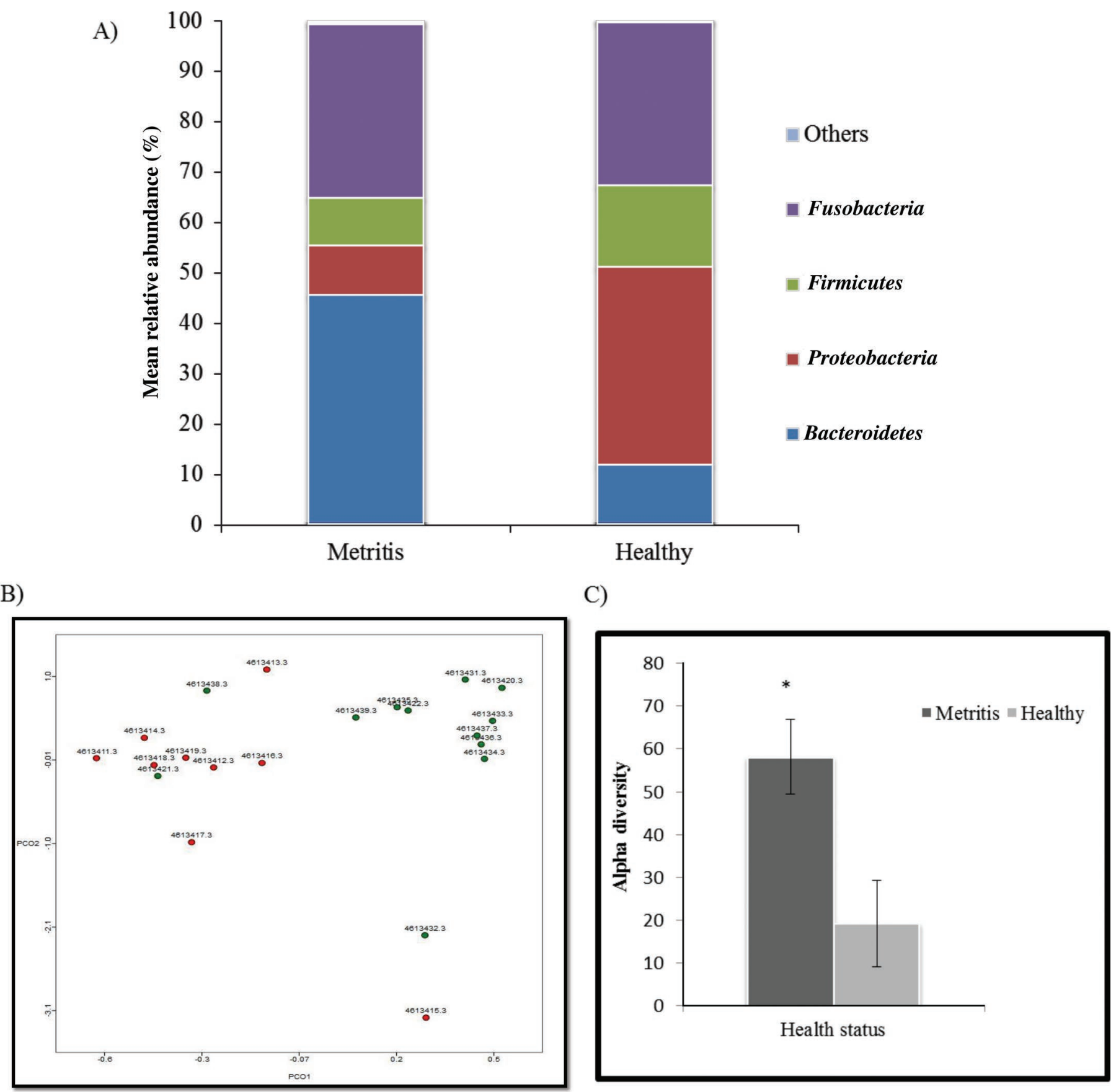

Figure 1. Comparison of the bacterial populations in uteri of cows with metritis versus healthy cows. (A) Histograms representing the overall relative distribution of bacteria at the phylum level between uterine samples from cows with metritis and healthy cows. Shading was assigned to phyla that constituted $>5.0 \%$ of all sequences observed within each group. (B) Principal component analysis based on taxonomic (phylum level) and functional distribution in each sample; red circles represent cows with metritis, and green circles healthy cows. (C) Sequence diversity for bacterial consortia for metritic cows and healthy cows (*diversity differed at $P<0.05$ ). Error bars represent SEM. Color version available online.

a healthy cow, were the ones with the highest numbers of observed phylotypes. This suggests that, in general, cows with metritis would be predicted to have highest number of distinct microbial species. Additionally, when accounting for taxon spatial arrangement (evenness), the higher value for the measure of diversity ( $\alpha$ diversity) was observed for the libraries from metritic cows (Figure 1, panel C). 


\section{Taxonomic Distribution in the Uterine Microbiome}

At the domain level, bacteria were the most abundant, with an average abundance of $>80 \%$, followed by eukaryotes (approximately 15\%). Viruses comprised $0.002 \%$ of the sequences, and the remainder included unassigned sequences and archaeal sequences (0.010\%).

\section{Phylogenetic Profile of Archaea and Viruses}

Archaea and viruses accounted for a very low percentage of the total community (0.010 and $0.002 \%$, respectively). The most common archaeal species found in the uteri of endometritic and healthy cows were Methanobrevibacter smithii and Methanobrevibacter ruminantium. On the other hand, bovine herpes viruses were found only in cows with metritis $(n=4)$. Because of their low prevalence, the Archaea and viruses were excluded from all disease association analyses.

\section{Phylogenetic Profile of Bacteria}

The relative distribution of phyla in the uterine samples is shown in Figure 1A, and the most abundant genera in both microbiotas are depicted in Figure 2. Sequences from the metritic uterine samples predominantly contained phylotypes affiliated with Bacteroidetes, Proteobacteria, Firmicutes, and Fusobacteria (Figure 1A). In both microbiotas, phyla with prevalences $<5.0 \%$ were combined and designated "Others." Among healthy cows, the same types of phyla were present as in metritic cows; however, the relative abundances were different. The proportion of Proteobacteria and Bacteroidetes in healthy cows accounted for approximately 40 and $10 \%$ of all bacteria, respectively, whereas in metritic cows, the percentage of Proteobacteria decreased to $10 \%$ and Bacteroidetes increased to more than $45 \%$. Interestingly, Fusobacteria were highly prevalent in both microbiotas, accounting for approximately $40 \%$ of the total bacterial community.

To derive interrelationships among uterine samples, we applied principal component analysis in the MGRAST analysis pipeline with its default statistical parameters, which further highlighted distinct differences between the healthy and metritic uterine microbiotas (Figure 1B). Most samples from cows of the same health status (i.e., healthy or metritic) tended to cluster together, suggesting they were more similar to each other compared with samples from cows having the other health status. Likewise, total sequence reads from cows with metritis accounted for $85.38 \%$ of the total number of $16 \mathrm{~S}$ rDNA sequence reads in the present study. The number of sequences affiliated to Fusobac- teria and Bacteroides were significantly higher in cows with metritis than in healthy cows (data not shown).

The most abundant bacterial taxa identified in the uterine microbiotas of metritic and healthy cows are depicted in Figure 2. Detailed analysis of uterine-sample genera revealed that both microbiotas had great abundance of Fusobacterium, averaging 29.4\% ( $\mathrm{SD} \pm 31.9)$ of all reads for metritic cows and $8 \%(\mathrm{SD} \pm 12.8)$ for healthy cows $(P<0.05$; Figure 2$)$. The increased relative abundance of Bacteroidetes in cows with metritis was driven mostly by greater abundances of Bacteroides $(15 \pm 18.3 \%$ vs. $6 \pm 10.7 \% ; P=0.3$; Figure 2$)$ and Porphyromonas $(24 \pm 32 \%$ vs. $0.34 \pm 0.44 \%$; $P<$ 0.05 , Figure 2). Large shifts in bacterial populations within Proteobacteria were driven by declines in relative abundances of Escherichia and Shigella in metritic cows (0.06 and $0.6 \%$, respectively) versus healthy cows (3.7 and $1.7 \%$, respectively; $P=0.38$; Figure 2 ). The relative abundances of Enterococcus, Staphylococcus, and Ureaplasma were low in both health statuses: approximately $0.2,0.1$, and $0.08 \%$, respectively, for cows with metritis, and $0.01,0.01$, and $0.008 \%$ for healthy cows.

\section{Functional Profile of the Uterine Microbial Community}

The SEED database arranges metabolic pathways into a hierarchical structure in which all of the genes required for a specific task are categorized into subsystems and classified into levels (Overbeek et al., 2014). Functional analysis at level 1 using the SEED database in MG-RAST revealed that, among the functional systems that were identified in bovine uteri, the most predominant, as expected, were basic functional genes. "Protein metabolism" accounted for the greatest number of coding sequences, followed by "carbohydrates" and "clustering-based subsystems" (systems that are independently grouped with no previous specific classification) (Figure 3A). These reads accounted for 9.3 to $16.1 \%$ of the total reads referred to the functional profile.

Figure 3B illustrates differential representation by mean proportion in the predicted level-1 functional subsystems that are statistically significantly different between the uterine microbiotas of metritic and healthy cows $(P<0.05$ and an effective size of 0.5 threshold in STAMP. These functional categories were (1) "membrane transport," (2) "virulence, disease and defense mechanisms," and (3) "stress response." Analysis of the microbiome composition based on level-2 classification of these 3 subsystems allowed finer resolution of the functional categories. 


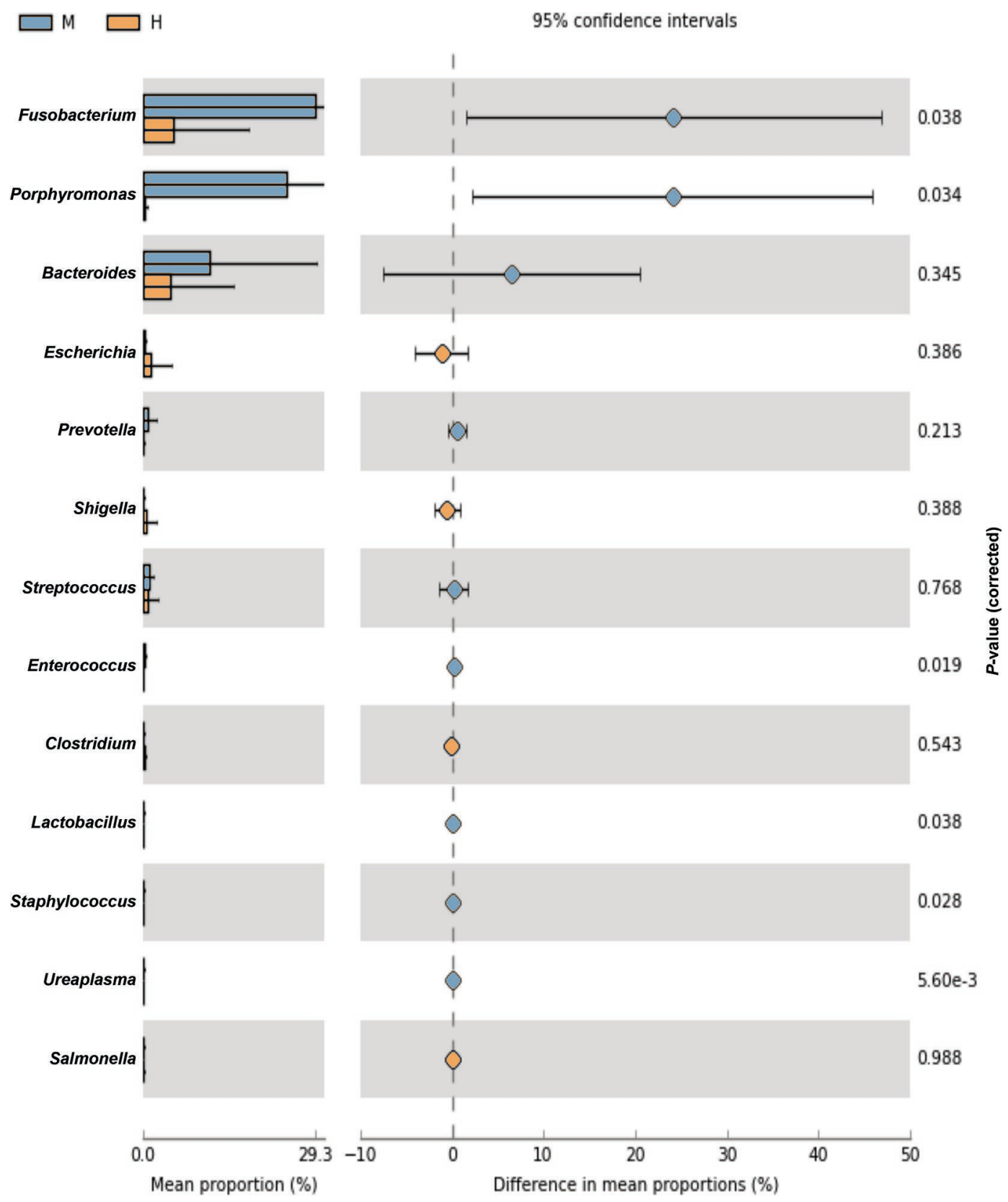

Figure 2. Extended error bar plots illustrating differential representation by mean proportion and their differences in bacterial genera common to metritic cows $(\mathrm{M})$ and healthy cows $(\mathrm{H})$, ranked by respective effect size of 0.5 thresholds in STAMP (Statistical Analysis of Metagenomic Profiles; http://kiwi.cs.dal.ca/Software/STAMP) and $P$-values. Genera with a $P$-value $<0.05$ were deemed statistically significant. Color version available online.

The relative abundances of genes coding for "membrane transport" functions are depicted in Figure 4A. Genes related to "protein translocation across the bacterial cytoplasmic membrane" and "protein secretion systems VI" predominated in the uterine microbiota of metritic cows.

Virulence-related genes included genes in the following subsystems: "adhesion," "bacteriocins and antibac- terial peptides," "detection," "invasion and intracellular resistance," "resistance to antibiotics and toxic compounds," and "toxins and superantigens" (Figure 4B). "Resistance to antibiotic and toxic compounds" was the predominant subsystem identified in the "virulence, disease, and defense" systems (>50\%) for both metritic and healthy microbiota. The "adhesion" subsystem, "bacteriocins," and "ribosomally synthesized antibac- 
terial peptides" were significantly overrepresented in the microbiota of healthy cows. Signal peptidase 1 was predominant within the "adhesion" subsystem. "Bacteriocins" and "synthesis of antibacterial peptides" were dominated by functions involved in tolerance to colicin E2 within the microbiota of healthy cows, which was unique to this group and represented $0.01 \%$ of the total number of sequences affiliated to functional profile. Sequences assigned to "toxins and superantigens" did not vary between the 2 health statuses.

Additionally, genes associated with bacterial responses to environmental stress are depicted in Figure
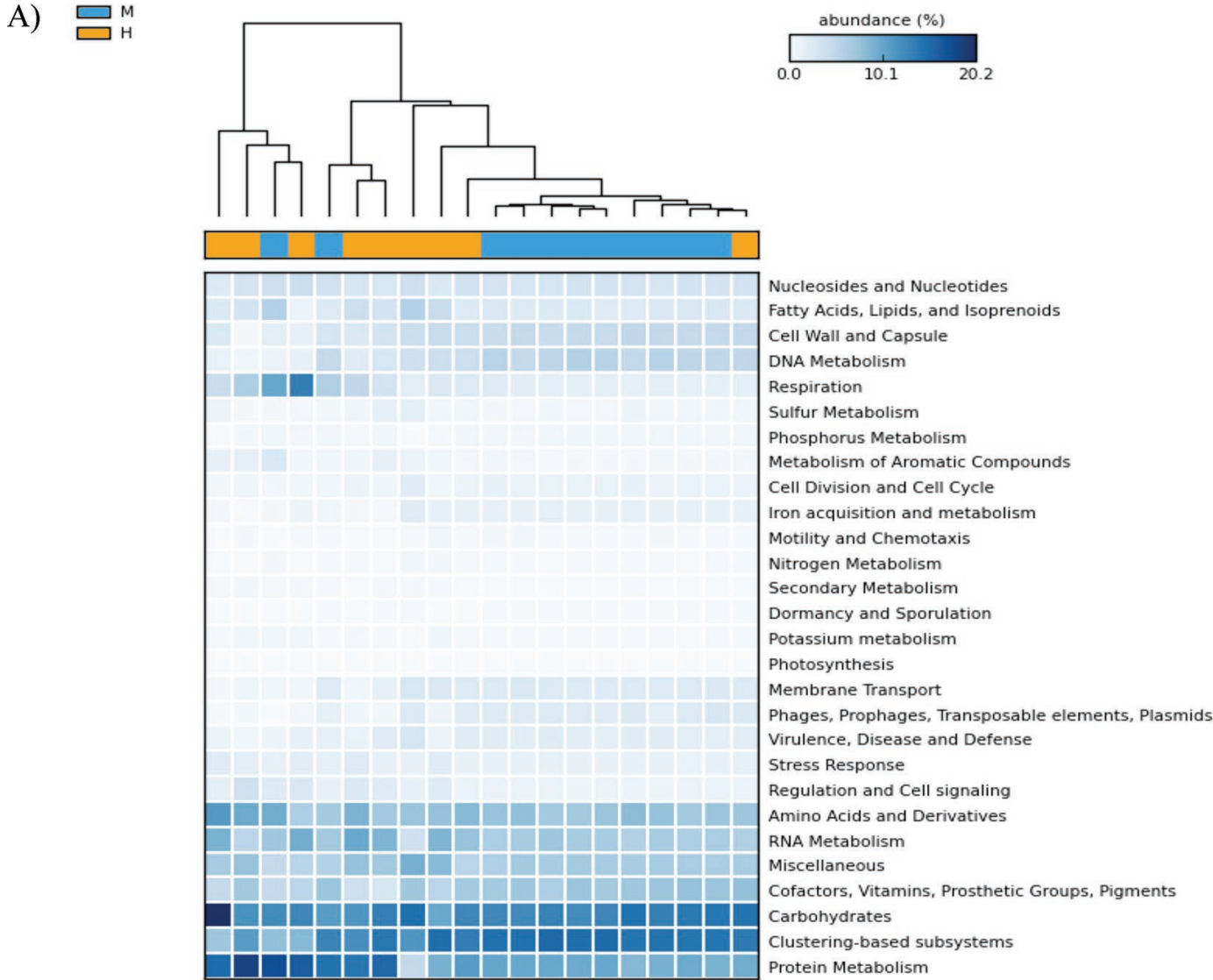
Cofactors, Vitamins, Prosthetic Groups, Pigments

d subsystems

rotein Metabolism

B)

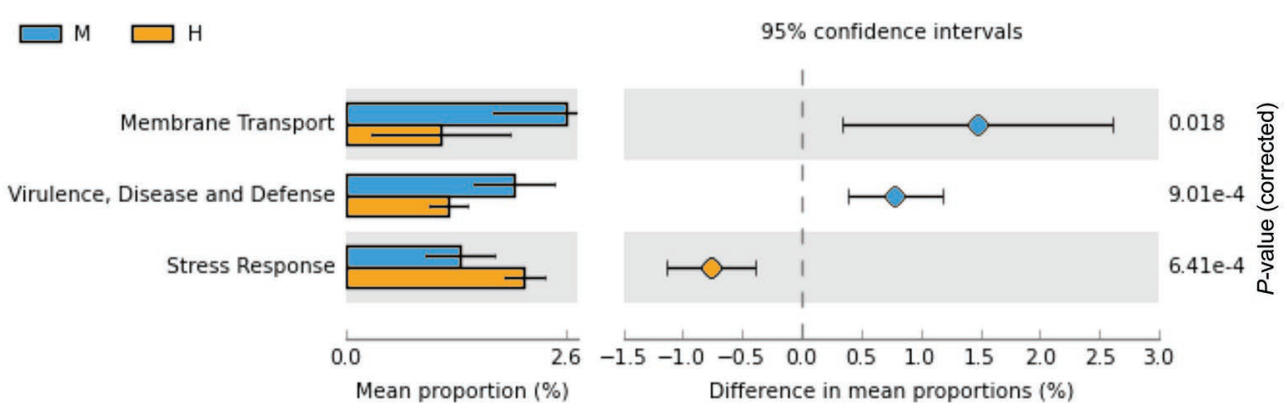

Figure 3. Distribution of the functional categories of uterine metagenomic data for metritic $(\mathrm{M})$ and healthy $(\mathrm{H})$ cows. The data were normalized in the MG-RAST (metagenomic rapid annotations using subsystems technology) pipeline. (A) Heatmap dendrogram showing functional categories at subsystem level 1 ranked by relative abundance. (B) Extended error bar plots representing abundance profiles of predicted functional gene categories that showed significant statistical differences between metritic cows and healthy cows. The results were filtered using a $P$-value of 0.05 and an effective size of 0.05 threshold in STAMP (Statistical Analysis of Metagenomic Profiles; http://kiwi.cs.dal.ca/Software/ STAMP). Color version available online. 
A) Membrane transport

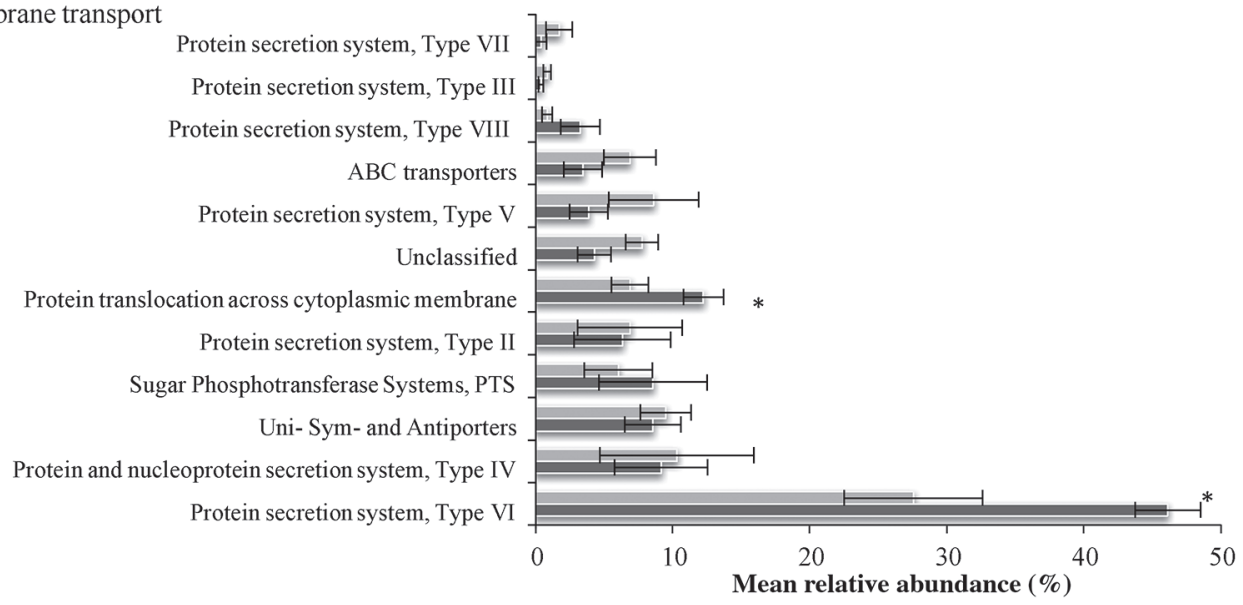

B) Virulence

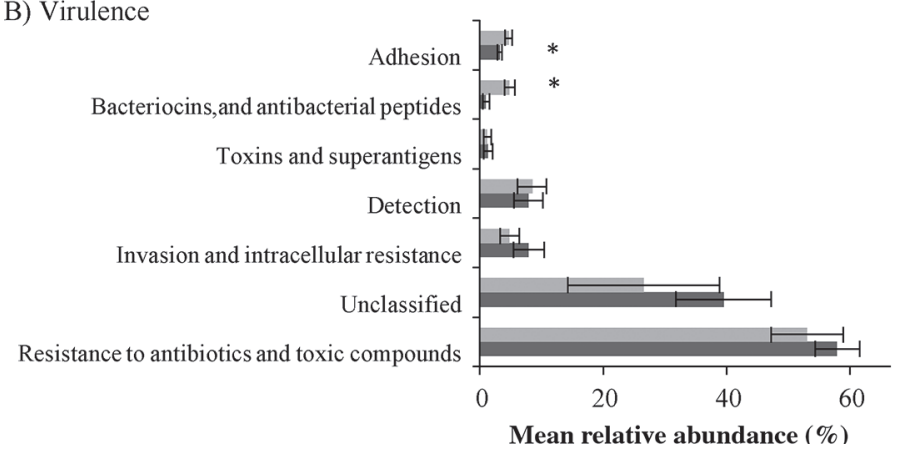

C) Stress

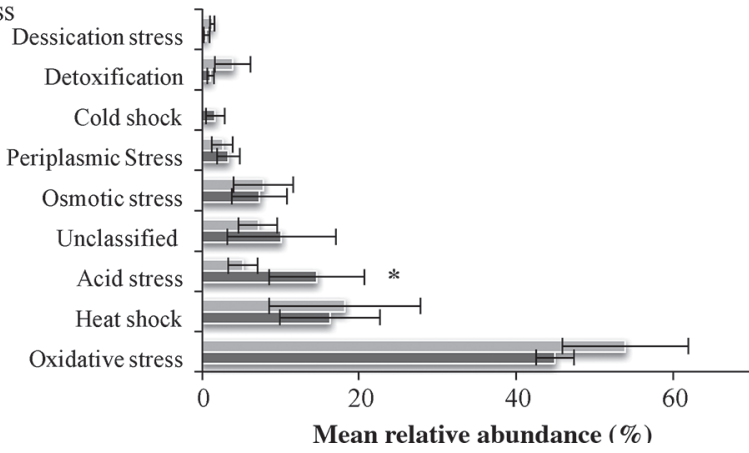

Figure 4. Statistical analysis from analysis of similarity (ANOSIM) pairwise comparisons of functional assignments at subsystem level 2 between metritic cows and healthy cows using Bray-Curtis values. The graphs depict the distribution of categories within DNA metabolism, membrane transport, stress response, and virulence subsystems at level 2 identified in both health statuses. The x-axis represents mean relative abundance and the error bars are the standard error of the mean. Dark gray bars represent cows with metritis and light gray bars represent healthy cows. (ANOSIM $* P \leq 0.05 ; \mathrm{R} \geq 0.5$ ). $\mathrm{R}$ values indicate the degree of functional genes separation across health status; values approaching unity indicate clear separation $(\mathrm{R}=0.5$ indicates dissimilarity with some overlap).

4C. "Cold shock" and "acid stress" were significantly increased in the microbiota of metritic cows, whereas "detoxification" and "oxidative stress" were highly represented in the microbiota of healthy cows. Sequences affiliated with resistance mechanisms to acid inside acid stress function were exclusive to metritis microbiota, representing $0.03 \%$ of the total number of sequences affiliated to functional profile enclosed by metritis. The unique and relative abundance of genes encoding for tolerance to colicin E2 and resistance mechanisms to acid within each peculiar microbiota are represented in Figure 5A and B, respectively.

Predicted virulence factors within the category "resistance to antibiotics and toxic compounds" are depicted in Figure 6. "Multidrug resistance efflux pumps" and the "multidrug efflux pump CmeABC operon" were found to be significantly more abundant in the uterine microbiota of healthy cows. On the other hand, "copper homeostasis" and "resistance to vancomycin tolerance locus" were found to be significantly more abundant in metritic cows. Streptothricin and erythromycin resistance and cadmium resistance were found to be unique to the microbiota of metritic cows (Figure 5).

\section{DISCUSSION}

To our knowledge, this is the first study to have used shotgun metagenomics to investigate the composition and potential functional profile of the uterine microbiome of metritic dairy cows compared with healthy dairy cows. We detected an increased abundance of bacteria within the phyla Fusobacteria and Bacteroidetes in metritic cows, supporting the potential role of these microbes in the pathogenesis of metritis. Furthermore, the uterine microbiome of metritic cows had significantly higher abundances of genes coding for protein transport across the cytoplasmic membrane and type VI bacterial secretion systems. In contrast, in the uterine microbiome of healthy cows, genes coding for adhesion functions, bacteriocins, and ribosomally 
synthesized antibacterial peptides were significantly more abundant.

The largest numbers of high-quality sequence reads belonged to the phyla Bacteroidetes, Proteobacteria, Firmicutes, Fusobacteria, Actinobacteria, and Tenericutes, regardless of uterine health status. The occurrence and dominance of these phyla is consistent with previous studies, which demonstrated that metritis occurs as a result of uterine contamination by diverse groups of pathogens (Miller et al., 2007; Santos et al., 2011; Santos and Bicalho, 2012; Jeon et al., 2015). Our results once again emphasize the importance of specific bacterial groups (e.g., Fusobacteria and Bacteroidetes) in metritis etiology. Indeed, we and others have pointed to these bacterial taxa as crucial for the development of metritis and bovine necrotic vulvovaginitis (Elad et al., 2004; Machado et al., 2012; Peng et al., 2013; Knudsen et al., 2015).

Although the uterine microbiota community has been shown to be a dynamic consortium of microbial species, many questions about cooperative interspecies signaling and antagonist interactions (e.g., antibiotic production) remain unanswered. Therefore, a more-direct approach for the study of such microbes has been to explore the molecular mechanisms behind the diversification of a physiologically coherent guild between metritic and healthy cows. Results from the MG-RAST pipeline analysis revealed that genes coding for functions associated with membrane transport, resistance to acid stress and with virulence, disease, and defense mechanisms were significantly more prevalent in the microbiota of metritic cows. However, within the "virulence, disease, and defense category," the presence of the antimicrobial peptide colicin E2 was uniquely found in the microbiota of healthy cows. The uterine microbiota of healthy cows also presented significantly higher abundances of genes associated with bacterial responses to environmental stress, especially oxidative stress.

Within the "membrane transport" category, higher abundances of genes coding for "protein translocation across the cytoplasmic membrane" and "secretion systems" were found in the microbiota of metritic cows. Protein secretion systems are sometimes referred to as puncturing devices that can target cell membranes to inject effector proteins. The importance of secreted proteins in bacterial pathogenesis has been previously
A)

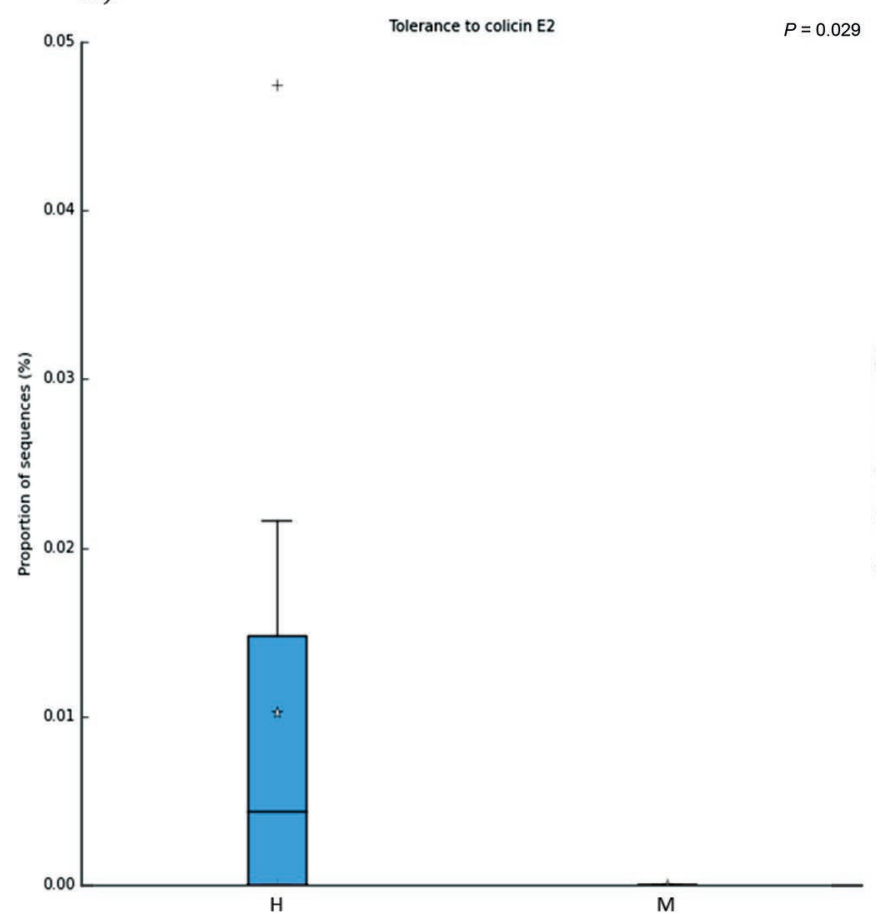

B)

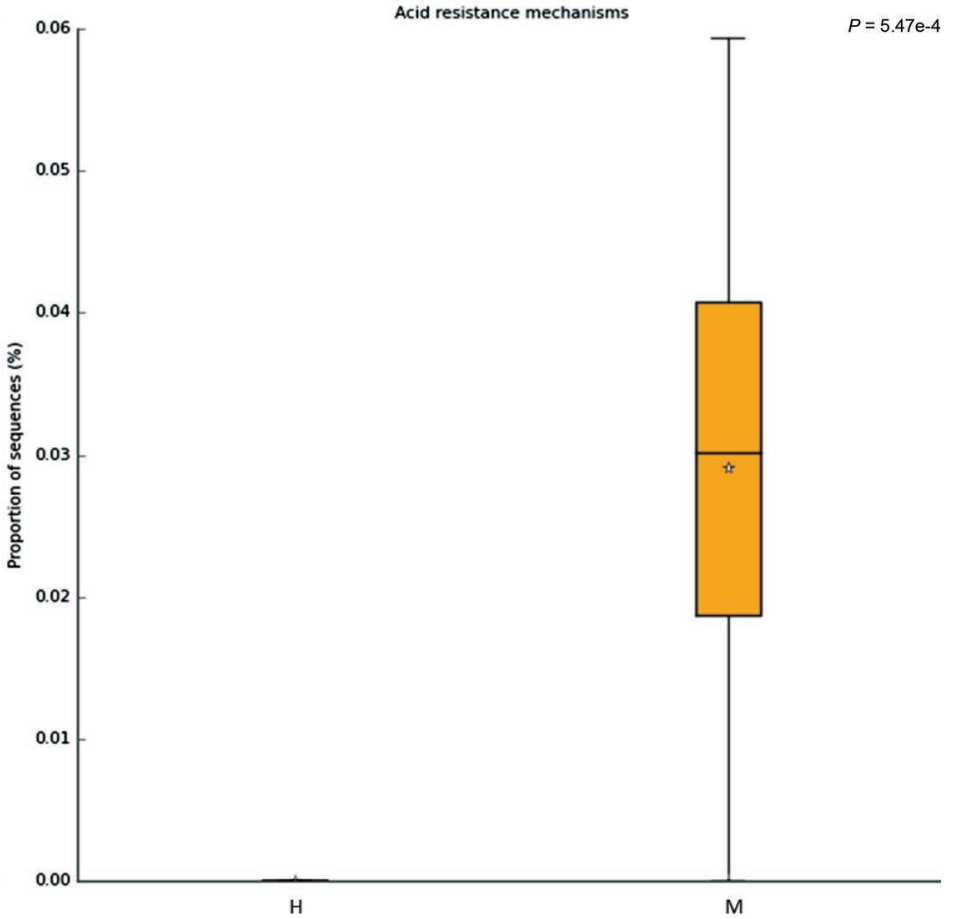

Figure 5. Box plot showing the distribution of the functional categories at subsystem level 2 of uterine metagenomic data for metritic (M) and healthy $(\mathrm{H})$ cows. The data were normalized in the MG-RAST (metagenomic rapid annotations using subsystems technology) pipeline and ranked by relative abundance using STAMP software (Statistical Analysis of Metagenomic Profiles; http://kiwi.cs.dal.ca/Software/STAMP). The unique presence and relative abundance of genes encoding for tolerance to colicin (healthy microbiota) and sequences affiliated to resistance mechanisms to acid (metritis microbiota) are represented in panels A and B, respectively. Boxes indicate the interquartile range (IQR; 75 th to 25 th of the data); the median value is shown as a line within the box, and the mean value as a star. Whiskers extend to the most extreme value within $1.5 \times$ IQR, and outliers are shown as crosses. Color version available online. 


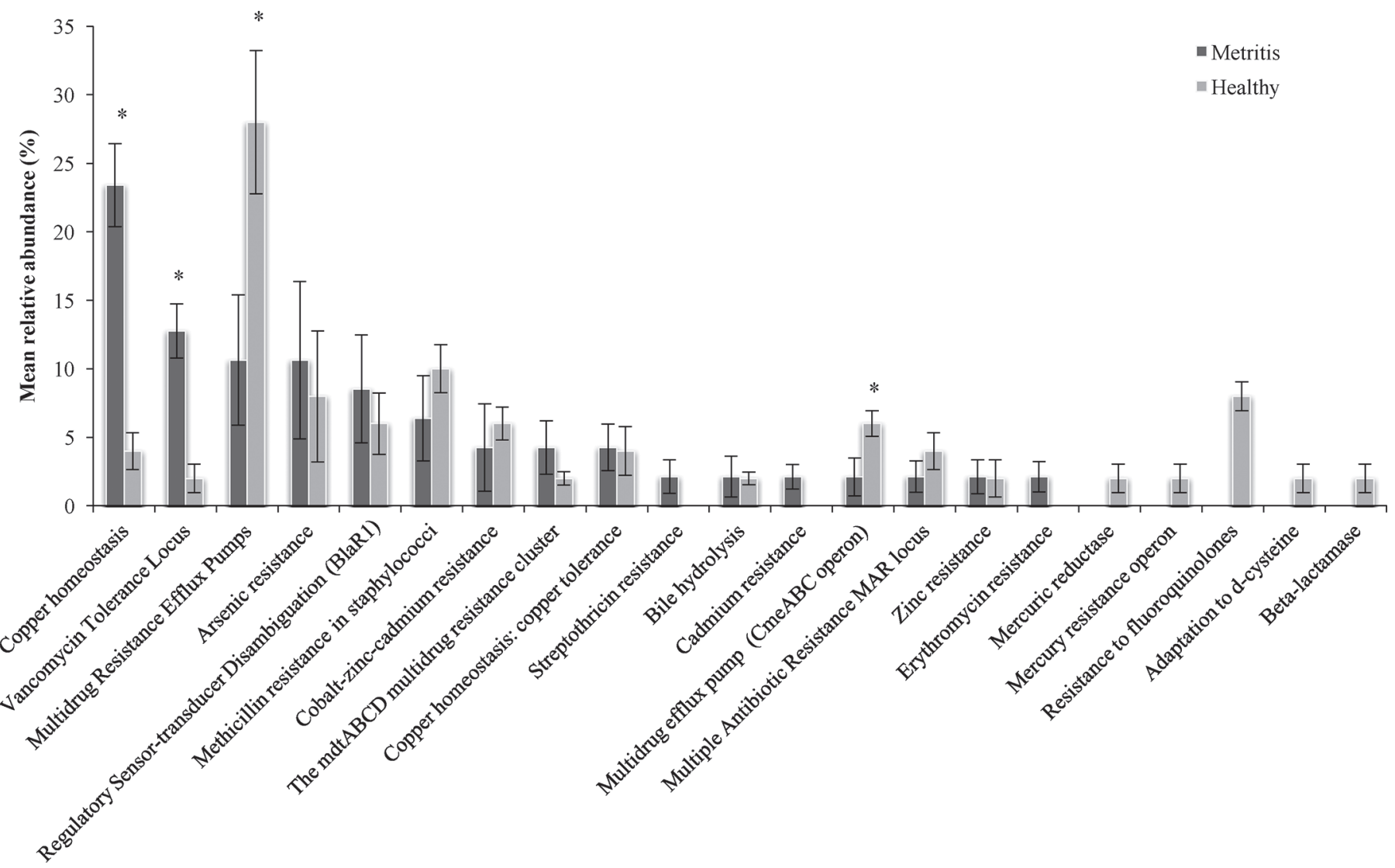

Figure 6. Relative abundance of virulence factors within the functional category "resistance to antibiotics and toxics compounds" in the uterine microbiota of dairy cows with metritis and healthy dairy cows. Error bars represent SEM. [Analysis of similarity (ANOSIM) $* P \leq 0.05$; $R \geq 0.5]$. $R$ values indicate the degree of functional genes separation across health status; values approaching unity indicate clear separation ( $R$ $=0.5$ indicates dissimilarity with some overlap).

documented, and these proteins have a wide range of biological functions (Driessen and Nouwen, 2008). Successful infection by bacteria requires manipulation of the host organism through 3 principal categories: bacterial adhesion, secretion of toxins into the extracellular milieu, and injection of virulence factors into the host cell (Lee and Schneewind, 2001). Therefore, it was not surprising to find that the microbiota of metritic cows express, at high levels, genes encoding factors involved in "protein translocation across membranes" and "secretion," processes that aid in uterine epithelial colonization and invasion of the mucosal surface by bacteria. Our findings corroborate earlier findings of Sheldon et al. (2010), who showed that endometrial pathogenic $E$. coli were more adherent and invasive against endometrial epithelial and stromal cells than E. coli isolated from clinically unaffected uteri.

The processes of protein translocation and secretion are heavily involved in adhesion to and infection of host cells mediated by gene products involved in the biosynthesis of fimbriae, flagella, outer membrane proteins, and lipopolysaccharides (Hueck, 1998; Morgan et al., 2004). Somewhat surprisingly, in the present study, virulence factor genes coding for "adhesion" functions and "bacteriocins and ribosomally synthesized antibacterial peptides" were significantly more abundant in the uterine microbiota of healthy cows compared with metritic cows. Genes coding for signal peptidase 1 were predominant within the "adhesion" function. Signal peptidases are distinct enzymes that cleave off extra peptide extensions of secretory proteins, enabling the mature proteins to cross the membrane and reach their target locations (Tuteja, 2005). Therefore, signal peptidases play a key role in the transport of proteins across membranes in all living organisms and would not be expected to be expressed differentially between the microbiotas of healthy and metritic uteri.

On the other hand, a distinctive finding of our study was the presence of genes coding for tolerance to colicin E2 uniquely in the uterine microbiota of healthy cows. Colicin E2 is an endonuclease that kills sensitive strains by causing rapid and extensive degradation of 
their DNA (Schaller and Nomura, 1976). The plasmid encoding colicin E2 was found to be present in the uterine microbiota of healthy cows; however, it is unknown whether colicin E2 production and resistance profiles arise independently, as there is a complex pleiotropy involved in generating colicin E2 resistance, including selection pressures that maintain the colicin E2 plasmid (Feldgarden et al., 1995).

The hypothesis of a potential role for colicin E2 during early intrauterine infection is supported by decades-old observations. For example, early experiments showed that colicin E2 production can be induced under both aerobic and anaerobic conditions, but E2-colicinogenic cell mass is sharply reduced under anaerobic conditions (Herschman and Helinski 1967). The involute uterus of the dairy cow is a naturally anaerobic environment, resulting from the combination of reduced uterine blood flow due to necrosis of the caruncles, and oxygen consumption by polymorphonuclear neutrophils fighting the heavy intrauterine contamination in the first days postpartum (El-Azab et al., 1988). This disparity in the rates of E2-colicinogenic bacterial growth may help explain how the intrauterine microbiota shifts rapidly during the first week postpartum. It seems that the presence of tolerant colicin E2 commensal bacteria within the uterine microbiota of healthy cows helps to prevent invasion of the uterus by bacterial species that disturb uterine homeostasis, causes dysbiosis, and favors the growth of strict gram-negative anaerobes, such as F. necrophorum, and Bacteroides spp.

Furthermore, colicin E2 activity is optimal at slightly acidic $\mathrm{pH}$ (6.4) and decreases at basic $\mathrm{pH}(7.3-7.6)$ (Herschman and Helinski, 1967), similar to neutrophil bactericidal activity (Reeves et al., 2003). Moreover, inflammatory conditions in cattle are reported to cause an increase in the $\mathrm{pH}$ of fluid excretions (Marschke and Kitchen, 1985; Cheong et al., 2012). These findings are consistent with our level-2 results, in which we found that genes involved in resistance to acid stress were exclusive to the microbiota of metritic cows, suggesting that microbial resistance to acid stress is important for pathogen survival in the early postpartum uterine environment.

Finally, genes encoding multidrug resistance efflux pumps, which have been previously linked to bacterial pathogenicity (Piddock, 2006), and heavy metal resistance (mercuric reductase and mercury resistance operon) were found in the uterine microbiota of healthy cows only, as were genes involved in resistance to fluoroquinolones, adaptation to D-cysteine (a powerful inhibitor of $E$. coli growth), and antibiotic resistance ( $\beta$-lactamase), consistent with the expression of antibiotics as potential mechanisms of homeostasis in the healthy microbiota (Duran-Pinedo et al., 2014). Some aerobic bacteria and archaea have evolved resistance mechanisms that function to degrade organic compounds; however, those functions result in lower organismal fitness (Nies, 2003). Therefore, the presence of significant bacterial overload coupled with lower oxygen availability due to increased neutrophil oxidative burst in necrotizing tissues may contribute to the perturbation of homeostasis in health-associated microbiota.

\section{CONCLUSIONS}

These results corroborate the existing literature, in that they confirm the potential role of bacteria within the phyla Fusobacteria and Bacteroidetes in the pathogenesis of metritis and provide new evidence that the presence of tolerant colicin E2 commensal bacteria within the uterine microbiota of healthy cows may help prevent invasion of the uterus by bacterial species that disturb uterine homeostasis and cause dysbiosis, inflammation, and infection. Additionally, genes coding for resistance to acid stress were found exclusively in metritic cows, suggesting that microbial resistance to acid stress is important for pathogen survival in the infected uterus. Finally, the microbiota of metritic cows expressed genes for protein translocation across membranes and secretion systems, which would aid in uterine epithelial colonization and invasion of the mucosal surface by pathogenic microbes.

\section{REFERENCES}

Bicalho, R. C., V. S. Machado, M. L. Bicalho, R. O. Gilbert, A. G. Teixeira, L. S. Caixeta, and R. V. Pereira. 2010. Molecular and epidemiological characterization of bovine intrauterine Escherichia coli. J. Dairy Sci. 93:5818-5830.

Bonnett, B. N., S. W. Martin, V. P. Gannon, R. B. Miller, and W. G. Etherington. 1991. Endometrial biopsy in Holstein-Friesian dairy cows. III. Bacteriological analysis and correlations with histological findings. Can. J. Vet. Res. 55:168-173.

Cheong, S. H., D. V. Nydam, K. N. Galvao, B. M. Crosier, A. Ricci, L. S. Caixeta, R. B. Sper, M. Fraga, and R. O. Gilbert. 2012. Use of reagent test strips for diagnosis of endometritis in dairy cows. Theriogenology 77:858-864.

Cole, J. R., Q. Wang, E. Cardenas, J. Fish, B. Chai, R. J. Farris, A. S. Kulam-Syed-Mohideen, D. M. McGarrell, T. Marsh, G. M. Garrity, and J. M. Tiedje. 2009. The ribosomal database project: Improved alignments and new tools for rRNA analysis. Nucleic Acids Res. 37:D141-D145.

Clarke, K. R., and R. N. Gorley. 2001. Primer v5: User manual/tutorial. Primer-E Ltd., Plymouth, UK.

DeSantis, T. Z., P. Hugenholtz, N. Larsen, M. Rojas, E. L. Brodie, K. Keller, T. Huber, D. Dalevi, P. Hu, and G. L. Andersen. 2006. Greengenes, a chimera-checked 16S rRNA gene database and workbench compatible with ARB. Appl. Environ. Microbiol. 72:5069-5072.

Driessen, A. J., and N. Nouwen. 2008. Protein translocation across the bacterial cytoplasmic membrane. Annu. Rev. Biochem. 77:643-667.

Drillich, M., O. Beetz, A. Pfutzner, M. Sabin, H. J. Sabin, P. Kutzer, H. Nattermann, and W. Heuwieser. 2001. Evaluation of a systemic antibiotic treatment of toxic puerperal metritis in dairy cows. J. Dairy Sci. 84:2010-2017. 
Duran-Pinedo, A. E., T. Chen, R. Teles, J. R. Starr, X. Wang, K. Krishnan, and J. Frias-Lopez. 2014. Community-wide transcriptome of the oral microbiome in subjects with and without periodontitis. ISME J. 8:1659-1672.

El-Azab, M. A., H. L. Whitmore, I. Kakoma, B. O. Brodie, D. J. McKenna, and B. K. Gustafsson. 1988. Evaluation of the uterine environment in experimental and spontaneous bovine metritis. Theriogenology 29:1327-1334. http://dx.doi.org/https://doi. org/10.1016/0093-691X(88)90012-X.

Elad, D., O. Friedgut, N. Alpert, Y. Stram, D. Lahav, D. Tiomkin, M. Avramson, K. Grinberg, and M. Bernstein. 2004. Bovine necrotic vulvovaginitis associated with Porphyromonas levii. Emerg. Infect. Dis. 10:505-507.

Feldgarden, M., S. Golden, H. Wilson, and M. A. Riley. 1995. Can phage defence maintain colicin plasmids in Escherichia coli? Microbiology 141:2977-2984.

Franzosa, E. A., X. C. Morgan, N. Segata, L. Waldron, J. Reyes, A. M. Earl, G. Giannoukos, M. R. Boylan, D. Ciulla, D. Gevers, J. Izard, W. S. Garrett, A. T. Chan, and C. Huttenhower. 2014. Relating the metatranscriptome and metagenome of the human gut. Proc. Natl. Acad. Sci. USA 111:E2329-E2338.

Hammer, Ø., D. A. T. Harper, and P. D. Ryan. 2001. Past: Paleontological statistics software package for education and data analysis. Palaeontol. Electronica 4:9.

Herschman, H. R., and D. R. Helinski. 1967. Purification and characterization of colicin E2 and colicin E3. J. Biol. Chem. 242:53605368.

Hilton, S. K., E. Castro-Nallar, M. Perez-Losada, I. Toma, T. A. McCaffrey, E. P. Hoffman, M. O. Siegel, G. L. Simon, W. E. Johnson, and K. A. Crandall. 2016. Metataxonomic and metagenomic approaches vs. culture-based techniques for clinical pathology. Front. Microbiol. 7:484.

Hueck, C. J. 1998. Type III protein secretion systems in bacterial pathogens of animals and plants. Microbiol. Mol. Biol. Rev. 62:379-433.

Jeon, S. J., A. Vieira-Neto, M. Gobikrushanth, R. Daetz, R. D. Mingoti, A. C. Parize, S. L. de Freitas, A. N. da Costa, R. C. Bicalho, S. Lima, K. C. Jeong, and K. N. Galvao. 2015. Uterine microbiota progression from calving until establishment of metritis in dairy cows. Appl. Environ. Microbiol. 81:6324-6332.

Knudsen, L. R., C. C. Karstrup, H. G. Pedersen, J. S. Agerholm, T. K. Jensen, and K. Klitgaard. 2015. Revisiting bovine pyometra-New insights into the disease using a culture-independent deep sequencing approach. Vet. Microbiol. 175:319-324.

Kuczynski, J., C. L. Lauber, W. A. Walters, L. W. Parfrey, J. C. Clemente, D. Gevers, and R. Knight. 2011. Experimental and analytical tools for studying the human microbiome. Nat. Rev. Genet. $13: 47-58$.

LeBlanc, S. J., T. Osawa, and J. Dubuc. 2011. Reproductive tract defense and disease in postpartum dairy cows. Theriogenology 76:1610-1618. http://dx.doi.org/https://doi.org/10.1016/j. theriogenology.2011.07.017.

Lee, V. T., and O. Schneewind. 2001. Protein secretion and the pathogenesis of bacterial infections. Genes Dev. 15:1725-1752.

Lima, F. S., A. Vieira-Neto, G. S. Vasconcellos, R. D. Mingoti, E. Karakaya, E. Sole, R. S. Bisinotto, N. Martinez, C. A. Risco, K. N. Galvao, and J. E. Santos. 2014. Efficacy of ampicillin trihydrate or ceftiofur hydrochloride for treatment of metritis and subsequent fertility in dairy cows. J. Dairy Sci. 97:5401-5414. http://dx.doi. org/https://doi.org/10.3168/jds.2013-7569.

Machado, V. S., G. Oikonomou, M. L. Bicalho, W. A. Knauer, R. Gilbert, and R. C. Bicalho. 2012. Investigation of postpartum dairy cows' uterine microbial diversity using metagenomic pyrosequencing of the 16S rRNA gene. Vet. Microbiol. 159:460-469.

Marschke, R. J., and B. J. Kitchen. 1985. Detection of bovine mastitis by bromothymol blue $\mathrm{pH}$ indicator test. J. Dairy Sci. 68:12631269.

Meyer, F., D. Paarmann, M. D'Souza, R. Olson, E. M. Glass, M. Kubal, T. Paczian, A. Rodriguez, R. Stevens, A. Wilke, J. Wilken- ing, and R. A. Edwards. 2008. The metagenomics RAST serverA public resource for the automatic phylogenetic and functional analysis of metagenomes. BMC Bioinformatics 19:386 https://doi. org/10.1186/1471-2105-9-386.

Miller, A. N., E. J. Williams, K. Sibley, S. Herath, E. A. Lane, J. Fishwick, D. M. Nash, A. N. Rycroft, H. Dobson, C. E. Bryant, and I. M. Sheldon. 2007. The effects of Arcanobacterium pyogenes on endometrial function in vitro, and on uterine and ovarian function in vivo. Theriogenology 68:972-980.

Morgan, E., J. D. Campbell, S. C. Rowe, J. Bispham, M. P. Stevens, A. J. Bowen, P. A. Barrow, D. J. Maskell, and T. S. Wallis. 2004. Identification of host-specific colonization factors of Salmonella enterica serovar Typhimurium. Mol. Microbiol. 54:994-1010.

Nies, D. H. 2003. Efflux-mediated heavy metal resistance in prokaryotes. FEMS Microbiol. Rev. 27:313-339.

Overbeek, R., R. Olson, G. D. Pusch, G. J. Olsen, J. J. Davis, T. Disz, R. A. Edwards, S. Gerdes, B. Parrello, M. Shukla, V. Vonstein, A. R. Wattam, F. Xia, and R. Stevens. 2014. The SEED and the Rapid Annotation of microbial genomes using Subsystems Technology (RAST). Nucleic Acids Res. 42:D206-D214.

Parks, D. H., G. W. Tyson, P. Hugenholtz, and R. G. Beiko. 2014. STAMP: statistical analysis of taxonomic and functional profiles. Bioinformatics 30:3123-3124.

Peng, Y., Y. Wang, S. Hang, and W. Zhu. 2013. Microbial diversity in uterus of healthy and metritic postpartum Holstein dairy cows. Folia Microbiol. (Praha) 58:593-600.

Piddock, L. J. 2006. Multidrug-resistance efflux pumps - Not just for resistance. Nat. Rev. Microbiol. 4:629-636.

Pruesse, E., C. Quast, K. Knittel, B. M. Fuchs, W. Ludwig, J. Peplies, and F. O. Glöckner. 2007. SILVA: A comprehensive online resource for quality checked and aligned ribosomal RNA sequence data compatible with ARB. Nucleic Acids Res. 35:7188-7196.

Reeves, E. P., M. Nagl, J. Godovac-Zimmermann, and A. W. Segal. 2003. Reassessment of the microbicidal activity of reactive oxygen species and hypochlorous acid with reference to the phagocytic vacuole of the neutrophil granulocyte. J. Med. Microbiol. $52: 643-651$.

Roossinck, M. J., D. P. Martin, and P. Roumagnac. 2015. Plant virus metagenomics: Advances in virus discovery. Phytopathology 105:716-727.

Santos, T. M., and R. C. Bicalho. 2012. Diversity and succession of bacterial communities in the uterine fluid of postpartum metritic, endometritic and healthy dairy cows. PLoS One 7:e53048.

Santos, T. M., R. O. Gilbert, and R. C. Bicalho. 2011. Metagenomic analysis of the uterine bacterial microbiota in healthy and metritic postpartum dairy cows. J. Dairy Sci. 94:291-302.

Schaller, K., and M. Nomura. 1976. Colicin E2 is DNA endonuclease. Proc. Natl. Acad. Sci. USA 73:3989-3993.

Sheldon, I. M., G. S. Lewis, S. LeBlanc, and R. O. Gilbert. 2006 Defining postpartum uterine disease in cattle. Theriogenology 65:1516-1530.

Sheldon, I. M., A. N. Rycroft, B. Dogan, M. Craven, J. J. Bromfield, A. Chandler, M. H. Roberts, S. B. Price, R. O. Gilbert, and K. W. Simpson. 2010. Specific strains of Escherichia coli are pathogenic for the endometrium of cattle and cause pelvic inflammatory disease in cattle and mice. PLoS One 5:e9192.

Stojkov, J., M. A. von Keyserlingk, J. N. Marchant-Forde, and D. M. Weary. 2015. Assessment of visceral pain associated with metritis in dairy cows. J. Dairy Sci. 98:5352-5361.

Tuteja, R. 2005. Type I signal peptidase: An overview. Arch. Biochem. Biophys. 441:107-111.

Wilke, A., T. Harrison, J. Wilkening, D. Field, E. M. Glass, N. Kyrpides, K. Mavrommatis, and F. Meyer. 2012. The M5nr: A novel non-redundant database containing protein sequences and annotations from multiple sources and associated tools. BMC Bioinformatics 13:141. 